\title{
LA PREDICACIÓN SECUNDARIA EN EL HABLA ORAL INFORMAL DE CHILE
}

\author{
Martin Hummel \\ (Karl-Franzens-Universität Graz) \\ martin.hummel@uni-graz.at
}

\begin{abstract}
Resumen
Partiendo de la hipótesis según la cual la conversión directa del adjetivo en adverbio (p. ej. Maria habla rápido) fue el mecanismo de formación de adverbios más importante en el latín hablado, opinamos que la conversión directa debería de mantenerse con mayor vigor en las variedades del español menos afectadas por las normas de la expresión escrita que tienden a imponer los adverbios en -mente (p. ej. Maria habla rápidamente). De hecho, la conversión directa es de lejos el principal mecanismo de formación de adverbios en el corpus del español informal hablado en Chile analizado aquí. En concreto, la predicación secundaria que nos ocupa aqui aparece como variante funcional que emerge de un continuo borroso de formas y funciones producido por el uso de formas adjetivales dentro del sintagma verbal.
\end{abstract}

PALABRAS CLAVE: adjetivo, adverbio, lengua hablada, diacronía, predicación secundaria.

\begin{abstract}
Starting from the diachronic hypothesis that direct conversion from adjective to adverb (e.g. Maria habla rápido) was the predominant mechanism of adverb formation in spoken Latin, this paper assumes that direct conversion should be still more frequent in varieties of nowadays Spanish which were less influenced by the norms of standard written expression. In fact, informal Chilean Spanish appears to prefer direct conversion to adverbs formed with the suffix - mente (e.g. Maria habla rápidamente). The inflectional use of adjectives in secondary predication (e.g. Maria habla contenta) seems to derive directly from the traditional use of adjectives within the verb phrase. Not surprisingly, the corpus of Chilean Spanish displays a fuzzy continuum of adjective and adverb functions in the verb phrase from which secondary predication emerges occasionally.

KEYWORDS: adjective, adverb, spoken language, diachrony, secondary predication.
\end{abstract}

\section{Introducción}

Dos mecanismos de formación de adverbios coexisten, compiten y colisionan en las lenguas románicas: la conversión directa del adjetivo en adverbio invariable (adjetivo adverbializado), como en Maria habla rápido, y la conversión indirecta del adjetivo en adverbio con el sufijo -mente, como en Maria habla rápidamente. Según la teoría formulada en Hummel 1999 y 2002, la conversión directa fue el mecanismo principal del latín hablado (latín vulgar). La preeminencia creciente del adverbio en -mente en el desarrollo ulterior de la lengua acompañó la implantación de la tradición escrita normativa en las grandes lenguas románicas en los espacios políticos de Francia, Italia, Portugal y España. La vinculación diacrónica de estos mecanismos a la tradición oral, en el caso de la conversión directa, y a la tradición escrita, en el caso de los adverbios en -mente, explica la variación diasistemática actual: en la lengua escrita estándar, los adjetivos adverbializados se encuentran marginados frente al adverbio en -mente canónico. En la variación diatópica, los espacios que sufrieron poco la influencia de la norma escrita conservaron el adjetivo adverbializado como meca- 
nismo principal o exclusivo: el rumano, el sardo, los dialectos del sur de Italia, el francés de Acadia y de Louisiana, y, bajo la forma de tendencias de uso, una inclinación más fuerte en los países del Nuevo Mundo hacia el uso de la conversión directa, como consecuencia de un menor impacto de la escolarización comparado con Europa. En la variación diafásica y diastrática, se observa una inclinación más fuerte a usar la conversión directa en el habla informal o inculta, sobre todo en los países hispano- o lusohablantes de América, donde este mecanismo suele predominar claramente en el habla informal o inculta. Del mismo modo, la frecuencia de los adjetivos adverbializados aumenta en las novelas que recurren al registro coloquial (Hummel, 2000:368-372 y, para el francés, Kofler, 2007).

De esta manera, la diacronía de los adverbios de manera fue marcada por la competencia de dos sistemas formales, uno monocategorial, otro bicategorial:

sistema monocategorial

atributo (adjetivo y adverbio)

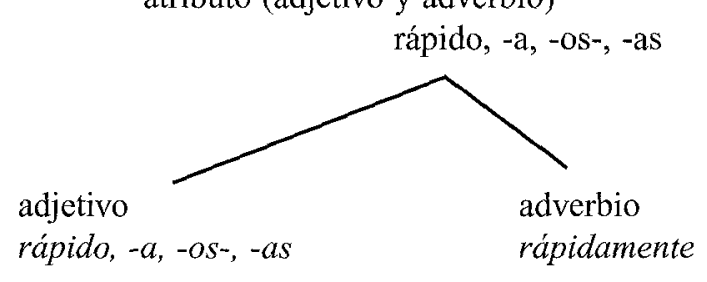

El sistema monocategorial, de tradición oral, usa una sola categoría morfológica, sin distinguir el adjetivo del adverbio a nivel de la lengua. Escogemos el término atributo para referirnos a esta categoría. En nuestra definición, son atributivas las relaciones en las que se atribuye a otra palabra una característica contenida en el significado de la palabra-atributo (casa bonita, cantar bonito, pensar bien, caminar tranquilamente, llegar contenta, terriblemente feo, etc.). En el sistema monocategorial, los morfemas de género y número son marcas ocasionales que pueden insertarse o no en una oración concreta, según las relaciones sintácticas y semánticas que el hablante desea establecer o marcar. Si la relación atributiva es adjetival en la sintaxis, el atributo tiende a ser flexionado (casa bonita, ella llega cansada), mientras que la flexión tiende a faltar siempre que la relación atributiva se dirige a un verbo (ella canta bonito) o a otro atributo (una chica medio tonta). Según hemos observado en otro lugar (Hummel, 2000, 2002), el sistema monocategorial del latín vulgar conformó un núcleo duro de adjetivos adverbializados, generalmente cortos y muy usuales, que sobrevivió en la lengua escrita al diluvio de los adverbios en -mente. Como consecuencia de ello, estos adjetivos adverbializados suelen ser vistos, en la gramaticografía marcada por la tradición escrita, como lista casi cerrada, poco productiva y algo más larga en América que en Europa, que incluye sintagmas como hablar alto, respirar hondo, etc.

El sistema bicategorial distingue morfológicamente el adjetivo del adverbio, reservando las marcas de género y número para el adjetivo, y el sufijo invariable -mente para el adverbio. En latín, las perífrasis modales con el ablativo mente del sustantivo mens eran poco usuales: "In no Latin author and at no time did mente replace to any significant degree the traditional Latin adverbial suffixes (Karlsson, 1981:44)". Es posible que algunos adverbios en -mente nacieran directamente en el latín hablado, pero los primeros testigos apuntan claramente hacia su origen culto, especialmente en el discurso de la Iglesia (prona mente facere, devota mente facere, etc.) o del derecho (buena mente factum, con la variante preposicional de 
buena miente en español antiguo) (v. Hummel, 2002b). Pensamos, pues, que la gran mayoría de los adverbios en -mente nació en la tradición escrita, infiltrándose paulatinamente en el habla oral, sobre todo culta, en un proceso de difusión de arriba (lengua culta) hacia abajo (lengua inculta), produciendo asimismo formas dobles como igual / igualmente o rápido 1 rápidamente con diferenciación semántica y pragmática más o menos fuerte, según los adverbios en cuestión. Dejamos a un lado, aquí, la discusión de si el morfema-mente es un sufijo o un morfema flexional (comp. Salazar García, 2007:311-313). Si fuera cierta la segunda opción, el sistema binario dejaría al hablante la alternativa de marcar flexionalmente el atributo como adjetivo o como adverbio, pero las fuertes tendencias de lexicalización de los adverbios en -mente ponen un serio problema, entre otros, a este punto de vista.

\section{Hipótesis, corpus y metodología}

Desde el punto de vista metodológico, suponemos que las variedades diasistemáticas actuales del español reflejan las tradiciones históricas, con la peculiaridad de haber conocido efectos de ósmosis específicos en función del impacto de los sistemas monocategorial y bicategorial. Si ello es así, la observación de las variedades actuales nos ayudará a reconstruir el decurso diacrónico. Nos parece particularmente importante el análisis del habla oral actual, en la medida en que ya no tenemos acceso directo al habla oral de épocas pasadas. Es posible que las variedades de hoy nos abran más puertas hacia el pasado que la búsqueda de reflejos de la oralidad espontánea en los textos escritos. Si es cierta la teoría que acabamos de esbozar, deberíamos observar una presencia más fuerte del sistema monocategorial en el habla oral informal frente a un impacto más importante de los adverbios en -mente en los textos escritos. Disponiendo ya de los datos de un gran corpus escrito de 22 novelas españolas (Hummel, 2000) y de otros datos (Hummel, 1998a, 1998b, 2002a), decidimos analizar un corpus oral que no perteneciera al habla culta marcada por la norma escrita.

El corpus que analizaremos aquí está compuesto por nueve entrevistas con mujeres de 17 a 58 años en Santiago de Chile, registradas en 1995 y transcritas por Bettina Kluge (parcialmente publicadas en Kluge, 2005a y b). Las personas son oriundas del sur de Chile, de donde migraron a Santiago para trabajar como empleadas domésticas internas que viven en la casa de los "patrones" ("puertas adentro"). Estas mujeres crecieron mayoritariamente en estructuras campesinas sin infraestructura moderna, muchas veces sin electricidad, agua corriente ni transporte público. Las entrevistas se hicieron en Santiago en el Instituto Luisa Cardijn, que ofrece cursos de formación y diplomas escolares especialmente destinados a las empleadas domésticas. El material lingüístico recogido no refleja directamente el uso dialectal del sur de Chile. Aunque nacieron en un ambiente dialectal campesino, las mujeres se adaptaron al estándar nacional a través de su formación escolar básica o media y, posteriormente, por su vida en la capital en casa de familias de clase alta. Así, una de las personas entrevistadas le dice a Kluge que no está hablando con ella como en su casa del sur, sino como suele hablar con los niños de sus "patrones" que deben aprender a hablar correctamente (Marta, 1361-1382). A pesar de ello, los textos pertenecen a un registro oral informal que se acerca a lo que podríamos denominar el estándar coloquial nacional popular. Teniendo en cuenta la importancia de los flujos migratorios hacia los grandes centros urbanos en toda América Latina, las personas entrevistadas responden a un tipo de biografía y de aprendizaje lingüístico muy representativos de las tendencias actuales de uso del español. 
Diacrónicamente, Chile pertenece a una zona mucho menos afectada por el influjo escolar que España, es decir, suponemos que la tradición oral sigue siendo más auténtica que en España, especialmente en el uso de los adjetivos adverbializados. Las entrevistas duraron entre 25 y 180 minutos, la mayoría de ellas entre 45 y 60 minutos. La parte del texto que corresponde a la producción lingüística de las empleadas suma unas 70.000 palabras aproximadamente. Para la transcripción se usan los símbolos siguientes:

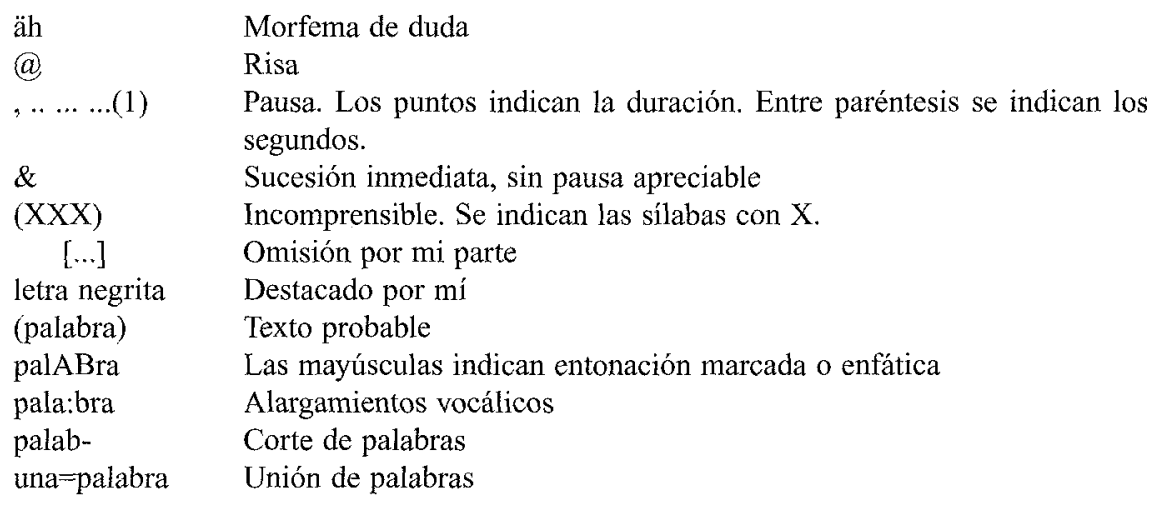

Las peculiaridades fonéticas fueron parcialmente transcritas, como, por ejemplo, la realización de /s/ como [h] o su elisión completa, la elisión de la /d/ intervocálica como en casá (= casada), etc. Los nombres de las personas son seudónimos. Los ejemplos se citan con el seudónimo seguido de la línea en la que empieza la cita, por ejemplo, (Julia, 345). "B:" introduce el discurso de la entrevistadora Kluge.

\section{Resultados anteriores}

Este artículo, que versa sobre la predicación secundaria, pertenece a una serie de trabajos en los que analizamos por separado distintas funciones atributivas en el mismo corpus: modificador del verbo (Hummel, 2007), modificador de otro atributo (adjetivo o adverbio) (Hummel en prensa a), adverbios de lugar y de tiempo (en prensa b), atributos enunciativos (en preparación), construcciones copulativas (en preparación).

En Hummel 2007, dedicado al estudio de los atributos directos (modificadores) del verbo en el Corpus Kluge, llegamos a confirmar la hipótesis de que los adjetivos adverbializados (conversión directa) se usan con mayor frecuencia como atributos de un verbo que los adverbios en -mente: la frecuencia de los adjetivos adverbializados es 8 veces superior a la de los adverbios en -mente, si se mira la frecuencia de las ocurrencias (token), y 5 veces superior, si se considera la frecuencia de los tipos (types). Contrariamente a lo que suelen escribir las gramáticas, los adverbios en -mente se usan principalmente como adverbios oracionales (62 ocurrencias, 9 tipos, por ejemplo, seguramente vendrá) y como atributo de un adjetivo (tipo terriblemente superior; 9 ocurrencias, 5 tipos) frente a sus 5 ocurrencias ( 5 tipos) como atributo de un verbo, como en hablar rápidamente. Si lo comparamos con las 40 ocurrencias de adjetivos adverbializados en función de atributo verbal, realizadas por 26 tipos diferentes, el uso del adverbio en -mente con la misma función resulta claramente minoritario en este 
corpus del habla informal. La comparación de estos datos con el corpus del español escrito constituido por 22 novelas (Hummel, 2000) pone de manifiesto las importantes diferencias que separan la tradición oral de la escrita. En el corpus literario la relación se invierte, en la medida en que se detectan 3774 ocurrencias de adverbios en -mente que funcionan como atributo de un verbo, mientras que las ocurrencias de los adjetivos adverbializados con la misma función se reducen a escasos 141 ejemplos (2000:366).

En Hummel (en prensa a) hemos tratado la atribución terciaria (atributos de adjetivos o adverbios), partiendo de la hipótesis de que el uso de los adjetivos adverbializados se vería dificultado siempre que abandonan la posición sintáctica junto al verbo, por la ausencia de una marca formal para la función adverbial (*alto importante), mientras que la movilidad de los adverbios en -mente quedaría favorecida por la marca formal del sufijo (altamente importante). El análisis del corpus nos obligó a rechazar nuestra hipótesis que daba la ventaja a los adverbios en - mente. De hecho, se observan muy pocos adverbios en -mente con funciones de atributo de adjetivo o de adverbio (11 ejemplares, 6 tipos), mientras que los adverbios del tipo tanto, mucho, cuanto, poco, bastante, todo, medio, etc., que comparten su forma con la del adjetivo, son numerosos, sobre todo si incluimos también los alomorfos tan, muy y cuan que se remontan históricamente a las formas plenas correspondientes. A diferencia de los atributos del verbo en el habla culta, donde los adjetivos adverbializados desaparecen en la mar de los adverbios en -mente, la función cuantitativa está claramente dominada por las formas del sistema monocategorial tanto en la expresión escrita culta como en la oral informal. Su movilidad oracional refuerza este hecho: poco importante, pagar poco, pocas casas. El inconveniente de la marca adverbial cero se ve compensado por el alto grado de lexicalización de la función cuantitativa en un paradigma de formas prácticamente limitado. Correlatos útiles de esta fijación léxica, los adverbios cuantitativos en -mente se constituyen en la parte dinámica y productiva de la función cuantitativa con formas como terriblemente, tremendamente, etc., que se usan también, aunque con frecuencia bastante limitada.

\section{Predicación secundaria: descripción funcional}

Los predicados secundarios pertenecen al grupo de los atributos indirectos que se refieren morfológica y semánticamente a un sustantivo en función de sujeto u objeto por e1 intermediario de un verbo al que pertenecen sintácticamente. Se distinguen básicamente ${ }^{1}$ los predicados secundarios dirigidos al sujeto del verbo (1) de los que se refieren a su objeto (2):

(1) María habla tranquila. ${ }^{2}$

(2) Carlos la encontró muerta.

Se trata de adjetivos flexionados que describen un estado mental o físico del participante sujeto u objeto desde la perspectiva del evento en el que estos participantes están involucrados. Así en (1), María está tranquila en el momento de hablar, y en (2), la persona está muerta cuando Carlos la descubre. En (1) el adjetivo describe el estado del sujeto que

1 Comp. los predicados secundarios orientados hacia el objeto indirecto del verbo, muy raros, en Seguí (1992:89) y Hummel (2000:164-166).

2 Los ejemplos en cursiva no provienen del Corpus Kluge. 
lo caracteriza durante el evento que el participante-sujeto protagoniza, en (2) la acción de Carlos le hace descubrir un participante con la característica expresada por el adjetivo (v. el análisis detallado en Hummel, 2000 y 2002, donde el lector encontrará también una bibliografía más completa). A diferencia de otros tipos de atribución, la predicación secundaria se manifiesta en nuestro corpus como esquema morfo-sintáctico bastante borroso. Al lado de ejemplos inequívocos $(\S 5)$, existen muchos ejemplos que tienden a confundirse con otras funciones atributivas.

\section{Los predicados secundarios 'auténticos'}

\subsection{Los predicados secundarios orientados al sujeto}

En ocho citas, la flexión funciona como expresión morfológica de la relación atributiva semántica que une el adjetivo al sujeto3:

(3) ella se vino y dehpué llegó ya: bien vestlda .. se llevó regalos a su mamá [...] como yo veía que lah niñah llegaban bien:+, vehtidah .. y yo- .. necesitaba=tener- .. Esas vestimentah también po (Marta, 364)

(4) y hay muchah- muchah amigah prefieren=Eso .. que no andar como=un- .. ellah ellas hacen todo: .. a: suh cosah pero, relajada tranquilah (Ráquel, 266)

(5) no, que uno tiene que ehtar, trabajando ahi encerrada encerrada .. äh, hahta que le toque su:, su sallda .. ya, y esah cosah algunah niñah no: ... no han soportAdo eso (Marta, 486)

(6) por eso yo+ cuando me vine acá me vine informada por mih tíah (Irina, 373)

(7) $\mathrm{Ah}$, yo=voy (que lo quiero) en diez año máh .. ser (todavía puertas afuera), no $\backslash .$. tener al menoh para vivir tranquila .. y:, no sé po, que mi hijo estU-, esté estudiando: + .. (Sandra, 1203)

(8) una hermana tuvo un hijo solte:ra (Sandra, 327)

(9) porque ya depué $((=$ después)) me ando media dormida (Marcela, 835)

(10) ehte año .. que termine (XX) este año y (que me deja ir) a las clase, tranquilat (Sandra, 818)

El morfema del género femenino - $a$ aparece claramente en los ejemplos. En el último ejemplo, tranquilo se refiere lógicamente al objeto del verbo dejar, pero la predicación secundaria se realiza dentro del sintagma ir tranquilo en el que tranquilo se refiere al agente implícito de $i r$, que corresponde al objeto me de dejar. Se trata, pues, de una predicación orientada hacia el sujeto implícito del verbo ir, a diferencia de casos del tipo la dejó dormi$d a$, donde el atributo se refiere directamente al objeto del verbo dejar. Notemos la irregularidad flexional de media dormida, donde medio recibe la marca del género femenino a pesar de su función adverbial. Hemos analizado el caso en Hummel (en prensa a), que participa de la misma dinámica que los fenómenos que trataremos aquí en el § 7. El ejemplo no entra aquí por la ocurrencia de medio, sino por la presencia del predicado secundario dormido.

3 Para el cálculo de las frecuencias hemos seguido la convención fijada en Hummel 2007 de contar como una sola ocurrencia el tipo bien, bien apagadita, mientras que la ocurrencia en dos sintagmas seguidos como en bien poco, bien poco cuenta como dos ocurrencias. 
Dos ejemplos, que no se encontrarían tan fácilmente en textos literarios, comprueban que la predicación secundaria es compatible con un núcleo predicativo copulativo:

(11) ahÍ yo pienso que cuando: .. äh ...(1) lah- lah niñas se quedan embarazadas jóveneh .. y ellas tienen la ilusión de casarse y lah dejan .. ya (Marta, 572)

(12) Estoy sentada descansada pero estoy trabajando con lah con lah manoh+, así (Marta, 971)

En estos ejemplos, las construcciones copulativas se quedan embarazadas y estoy senta$d a$ tienen como adjunto libre un predicado secundario orientado hacia el sujeto.

\subsection{Los predicados secundarios orientados hacia el objeto}

En cuatro ejemplos del corpus, los atributos flexionados se refieren al objeto directo del verbo:

(13) no sÉ, yo no me-, yo creo que sí, porque también a vece hablan: .. no sé, n:-=no, no dicen bien lah cosah [...] no la dicen:, tan:, [exact(ah)] (Marcela, 1067)

(14) sí .. en lo que sÍ me he fijado de no hablar lah palabrah, äh .. recortada, osea .. a decir la palabra correcta osea tratar de decir tOda la calabra (Irina, 249)

(15) hay que limpiA:r, la cocl:na .. azulEjos, lavar la loza por supuesto: .. äh:, loh bAño li-, bien, limpios\& (Sandra, 1078)

(16) no iba a volver a mi campo .. donde mi familia, porque ... nUnca me habían querido mu:cho-- .. y menos embarazada- $\backslash$ (Sandra, 692)

Aunque el escaso número de ejemplos no permita confirmarlo con seguridad, parece que el habla oral informal utiliza la predicación secundaria orientada hacia el objeto con mayor libertad que en nuestro corpus de 22 novelas españolas contemporáneas, en las que se observa una lista prácticamente cerrada de verbos que aceptan predicados secundarios orientados hacia el objeto (Hummel, 2000:153-154). Notemos en este contexto el uso resultativo del verbo limpiar, y quizá también de hablar y decir.

\subsection{Los predicados secundarios con verbos reflexivos}

El corpus contiene dos predicados secundarios que acompañan a un verbo reflexivo (en sentido largo, es decir, un verbo con se):

(17) había mucho barro en el invierno y en el verano mucho polvo, se llenAba de tierra uno+, había que bañarse entera con ropa y todo en la casa cuando (uno) llegaba (Marta, 191)

(18) es preciOso tenemoh vihta al: lago rANco/ .. que se ve lindísimo (Ráquel, 75)

Debido a la correferencialidad de sujeto y objeto en las construcciones reflexivas, el adjetivo del primer ejemplo puede ser interpretado como atributo del sujeto o del objeto (expresado por el morfema reflexivo). En el segundo ejemplo, la pasiva refleja implica inferencialmente un participante cognitivo que ve el lago Ranco, con lo cual el adjetivo lindísimo 
se refiere lógicamente al objeto del verbo ver, ya que el lago no tiene la capacidad de verse a sí mismo. Puestos aparte los problemas de interpretación semántica, que puede orientar la atribución más hacia el sujeto, como en Juana se marchó contenta, o hacia el objeto, como en Juana se ve feliz, la construcción es la misma que en 5.1. y 5.2.

\section{Los predicados secundarios 'semánticos'}

\subsection{La borrosidad sistémica}

No siempre es posible separar la predicación secundaria de los adjetivos adverbializados desde el punto de vista formal. La invariabilidad, que constituye la única marca morfológica del adjetivo adverbializado, no es una marca suficiente para distinguirlo de un adjetivo auténtico cuando el sustantivo al que atribuye su significado léxico viene en masculino singular:

(19a) María habla contenta.

(19b) Juan habla contento.

A diferencia de (19a), la oración (19b) es formalmente ambigua, aunque semánticamente también se trata de un predicado secundario, o sea, los hablantes cultos lo interpretarán así. De hecho, sólo la conmutación de (19a) con (19b) nos permite identificar el carácter flexional de contento en (19b).

En otro tipo de adjetivo, sólo la marca del plural es capaz de aclarar formalmente la relación atributiva:

(20a) María habla feliz.

(20b) Las mujeres hablan felices.

En los ejemplos siguientes de nuestro corpus, la duda se debe al hecho de que los adjetivos en $-e$ no tienen marca de género:

(21) me gusta sali:r andar li:bre, no eh que polole:an otrah que-, bueno que vitrinean a vece (Adela, 195)

(22) sí- ... así como llueve mucho y- .. me guhtó má el cllma porque an-, uno anda máh .. máh como libre de andar, con tanta ropa encima (Adela, 268)

(23) yo felÍ $((=$ feliz)), me iria a vivir al campo porque me encanta todo lo que eh ca:mpo (Sandra, 647)

(24) Pero:, si tuvlEra para vivir en el campo no digo .. RIca äh, (no tanto) mucho .. pero para vivir normaL/.. fellZ iría (Sandra, 1048)

(25) mi hermana mayor también es soltera, ya (los) treinta y cuatro años/ .. y (yo bueno) es que: .. yo feliz la tendria en mi casa, pero (es=que), o=sea si (yo me estuviera) a casar (y esto) viejital, yo feliz (te tengo) en mi casa, pero:+ .. a lo mejor no le va a gustar a mi esPOso .. y yo voy, no me va a peliar con ÉL (Julia, 810)

Son casos de ambigüedad debidos al sistema de la lengua y que también se dan en el uso normativo. El contexto excluye la interpretación de andar como verbo copulativo en (21) y (22). El segundo ejemplo pone de manifiesto la difícil delimitación de las unidades sintácti- 
cas en el habla oral, en la medida en que interviene un marcador de duda máh, máh seguido de una reformulación que confiere un estatus ambiguo al atributo libre. Notemos que la frecuencia de uso de los predicados secundarios en posición preverbal es relativamente alta, si la comparamos con el uso de adjetivos preverbales en textos literarios (Hummel, 2000:141, 145-148 y 408), con la posible excepción de poemas y canciones, donde el uso preverbal también es algo más frecuente. Si añadimos las ocurrencias calladito obedecer y yo sola proseguir que aparecen en los ejemplos (31) y (40), llegamos a 6 casos de anteposición, sin contar el caso ambiguo (22). Obsérvese, además, que la anteposición puede favorecer la invariabilidad, en la medida en que la función sintáctica que se refiere al participante, con sus posibles marcas de género y número, viene después.

En todos estos casos, la predicación secundaria no existe como categoría morfo-sintáctica, sino como hecho sintáctico-semántico que se infiere a partir del significado de las unidades en el enunciado. En andar libre tenemos que decidir por inferencia si es más fuerte la vinculación atributiva con el verbo o con el agente del verbo, sin que se pueda decir que la carga atributiva caiga en una sola función sintáctica.

Así pues, la predicación secundaria en español se asemeja parcialmente a la que se observa en alemán y en inglés, en la medida en que el inglés no tiene marcas morfológicas adjetivales de género y número, y que el alemán mantiene la invariabilidad siempre que el atributo es parte del sintagma verbal. El hablante alemán no tiene la posibilidad, como en español, de orientar la atribución optando, sea por la flexión, sea por la invariabilidad. La misma situación se produce en español cuando el sujeto tiene la forma del masculino singular:

masculino singular femenino singular plural

\author{
Hans schläft ruhig. \\ Maria schläft ruhig. \\ Hans und Maria schlafen ruhig.
}

Juan duerme tranquilo.

Maria duerme tranquila.

Juan y Maria duermen tranquilos.

\subsection{La borrosidad opcional}

La costumbre de reducir la [s] final a [h] o [ø] tiende a ocultar el morfema de plural en el habla oral chilena y aumenta asimismo el número de ejemplos ambiguos:

(26) Los chicos hablan contento ${ }^{(\mathrm{h})}$

No obstante, la elisión del morfema de número no se da en todos los casos. A diferencia de la borrosidad sistémica inevitable en 6.1., la ausencia del morfema de plural es una simple opción, puesto que los hablantes son perfectamente capaces de usarlo si lo desean. De cierta forma, el uso del morfema de plural sigue la misma pauta de inserción espontánea que los morfemas de género. Resulta interesante observar que la libertad de escoger o no las marcas de género y de número va más allá de la simple elisión fonética del morfema de plural, ya que también afecta al uso del morfema de género marcado (femenino), aunque en menor grado. En el ejemplo siguiente, no hay congruencia del atributo vestido ni con el plural llegan ni con el género y número del sujeto gente:

(27) yo creo que cada persona que llEga a una ciudad grande, debe adaptarse a la ciudad, porque $=\mathrm{eh}=\mathrm{lo}-1$ : la gente que viene de temuco, hay gente que llegan vehtido y $\mathrm{y}$ :, Ilevan suh culturah (Adela, 744) 
No es imposible que el atributo se encuentre en acuerdo morfológico con el verbo llegan vestido(s) por la costumbre de la elisión. Pero aun así, el adjetivo no corresponde en género al sujeto gente. Por eso, podríamos optar por una interpretación como adjetivo adverbializado invariable. Recuérdese, sin embargo, que en el ejemplo (3) aparecen las predicaciones secundarias llega vestida y llegan vestidas, y que el uso de predicados secundarios flexionados después del verbo llegar es muy usual en todos los registros de la lengua española. La prueba de conmutación apunta, pues, hacia la predicación secundaria. Por eso, podría tratarse de un predicado secundario 'semántico' como los de 6.1., con la diferencia de que, en este caso, la hablante renuncia a las marcas de género y número porque no le parece necesario al entendimiento del enunciado. Lo más importante, sin embargo, es observar que los tres ejemplos del sintagma llegar vestido comprueban el carácter espontáneo, enunciativo de la flexión, como es de esperar en un sistema monocategorial. Además, en nuestra muestra del habla oral informal semiculta, la lógica flexional no es la misma que en las gramáticas. De hecho, las gramáticas imponen una lógica de flexión de orden estructural intraoracional, con pocas excepciones, como la mayoría son, por ejemplo. El habla oral espontánea resta más importancia a las relaciones semánticas que se infieren al partir del sentido del enunciado (comp. Hummel, 2000:413-414). En nuestro ejemplo, la hablante escoge el acuerdo ad sensum de la forma verbal llegan con la gente, y posiblemente otro acuerdo ad sensum con las implicaciones genéricas de la gente, cuando opta por vehtido.

El corpus contiene también un ejemplo, en el que no hay concordancia en la predicación secundaria orientada hacia el objeto:

(28) mih hermanoh $[\ldots]$ siempre me dan un-, un pedacito para sembrar [...], y hay doh=hermanoh máh que viven aqui en santiago $\mathrm{y}=$ elloh tienen su casa $\mathrm{y}=\mathrm{ya} / . .$. tienen familia .. $y=a=e l l o h$ también les dan: .. pero ya leh dan $\operatorname{cosech} A: d o$.. cuando van:, a veraneAR- . . leh dan .. pApah le=dan=harina crUda .. traen huevI:tos, traen pollito de todo .. ya/ (Marta, 224)

En este ejemplo, el atributo cosechado viene bastante antes del objeto papas, harina cruda. Esto facilita sin duda la invariabilidad, aunque tampoco podemos excluir en nuestro corpus que cosechado equivalga a cosechados por la costumbre chilena de quitar la $-s$ final. Aun así, el ejemplo no marca la relación atributiva, dejando la interpretación de donde cae la carga atributiva al libre criterio inferencial del oyente. Otra posibilidad sería interpretar les dan cosechado como predicado secundario genérico invariable que luego es especificado por papas, harina cruda, etc.

En el siguiente ejemplo del corpus, podría tratarse perfectamente de un uso adverbializado de moderno:

(29) B: hm, ya .. y:, piensah que:, lah personah que, llegan de provincia a ehtablecerse en santiago .. deberían mantener suh rahgo, caracteríhticoh como:, su forma de vehtirse suh comidah y su forma de hablar .. o:, sería mejor, adaptarse al tiro

I: no, yo creo que no tanto como eso sino que, .. no copea, copear, o: o ve, hacer cosah que, hacen loh demá .. sino, de: .. de hablar bien, lo correcto

B: hm

I: $y$, creo que la la comida del:, del campo e excelente .. que no la cambian\&

B

\&ya .. y la ropa 
I: la ropa sí

B: (a)

I: que se vihtan má moderno a la moda .. eso sí e importante (Irina, 413)

No obstante, la costumbre de quitar la $-s$ final también nos permite interpretar el ejemplo como predicación secundaria se vistan más modernos, pero sólo si admitimos otra vez que la hablante le da una interpretación genérica al sustantivo femenino las personas. El sustantivo explícito las personas, al que podría referirse el verbo en plural se vistan, aparece algunas líneas antes en la pregunta de la interlocutora. Como se ve por el verbo vistan en plural, la persona entrevistada mantiene esta marca.

Hay dos ejemplos más en los que resulta imposible separar los adjetivos adverbializados de los adjetivos en predicación secundaria:

(30) B: da-, le dirías, a:, otros jóvenes de tu región/, que piensan en irse a vivir acá-- ...(1) qué deberían hacer/, qué NO deberían hacer-l

J: No sé, que: .. que se vengan pero: así como: .. que lo tomen tODo como relajadito (Julia, 1209)

(31) loh papá:=äh, decían algo ya había que obedecer .. ya+ .. y no había que contehTAr .. ya .. decir Sí +. ya [...] Y y:, calladito obedecer ..ya (Marta, 1229)

Podríamos aplicar criterios semánticos donde nos abandonan los criterios formales. En (31), el atributo es un participio que expresa el estado mental de las personas. Se trataría, pues, de un típico ejemplo de predicación secundaria. Por otro lado, no es imposible interpretar el atributo como adverbio que se refiere a la manera de actuar de los jóvenes. Empezaríamos una discusión sin fin con ejemplos y contraejemplos. La solución es otra: se deja la interpretación del alcance atributivo al criterio del oyente.

\section{La predicación secundaria 'falsa'}

Al lado de las tendencias sistemática (6.1) y opcional (6.2) hacia la invariabilidad y, por ende, a la atribución no dirigida formalmente, existe una tercera hacia la flexión 'ilógica', es decir, no justificada semánticamente:

(32a) vamos directos a casa

(32b) ventanas medias abiertas

(32c) la chica está toda contenta

(32d) me voy rapidita a coger el bus

La flexión marca formalmente los atributos como adjetivos, pero el sentido es adverbial. Podría hablarse con González García de adverbios flexivos (1997:310; comp. Hummel, 2000:409-412 y en prensa a), en los que podría incluirse quizás también el caso de vamos juntos, que trataremos infra en $\$ 8$. La norma culta tacha ejemplos como éstos de 'erráticos', 'vacilantes', 'ilógicos' o, con aparente neutralidad, de 'agramaticales'.

En un ejemplo del corpus, los predicados secundarios tranquilas y relajadas se refieren morfológicamente al objeto las cosas y lógicamente (por inferencia) al sujeto en singular: 
(33) asi que estoy:, sola durante el día, claro asi hago las cosas más tranquilas, más relajadas (Graciela, 361)

Se trata, pues, de una confusión adicional de orientación hacia el sujeto o el objeto. Comparemos el ejemplo con otro ya citado, en el que se repite casi literalmente el contexto del ejemplo que acabamos de ver:

(34) y hay muchah- muchah amigah prefieren $=$ Eso .. que no andar como=un- .. ellah ellas hacen todo: .. a: suh cosah pero, relajada tranquilah (Ráquel, 266)

Desde el punto de vista formal, los adjetivos relajadas y tranquilas pueden referirse al sujeto ellas o al objeto sus cosas, como en el ejemplo citado antes. En el segundo ejemplo, sin embargo, el sentido del enunciado nos hace optar por una relación atributiva con el sujeto ellas.

Firmadita en el ejemplo siguiente tampoco tiene justificación semántica:

(35) Ahora va a cumplir el año .. hm, ya tiene loh loh doce: .. mese, el año .. pero el veinte/ .. de ehte mes, cumple el año. [...] Sí, exquisita .. camina firmadita ... ya, entonces tengo que hacerle la comida yo también (Marta, 918)

El adjetivo firmadita es probablemente una forma abreviada de afirmadita. Podríamos clasificar el ejemplo como predicación secundaria, alegando que el adjetivo se refiere a la seguridad de la niña que aprende a andar. Preferimos pensar, sin embargo, que se trata de un adjetivo adverbializado flexionado a fines expresivos o lúdicos, como en la cita siguiente recogida en España: "Me voy rapidita que debo coger el bus" (Hummel, 2000:374). En los dos casos, el atributivo conlleva un morfema diminutivo que tiene el acento tónico. Los diminutivos son elementos expresivos, sobre todo cuando se usan con adjetivos y adverbios en los que la función de minoración no tiene sentido, con lo cual se transforman en elementos de intensificación. La flexión según el género aumenta el valor expresivo del diminutivo. De todas formas, sea cual fuere la interpretación, la flexión no es simplemente errática sino algo intencionada. A diferencia del ejemplo calladito obedecer, en el que se manifiesta una tendencia hacia el uso invariable, el ejemplo camina firmadita apunta hacia una costumbre opuesta.

\section{Borrosidad y lexicalización: solo y junto}

Existen casos en los que las tres tendencias observadas en $\S 5-7$ se manifiestan de forma lexicalizada dentro de un solo lexema polisémico y polifuncional. Profundizaremos el tema a la hora de tratar los adverbios oracionales como igual / igualmente, claro / claramente, bueno / buenamente, etc. Analizaremos aquí el caso de solo y junto que admiten, en algunos casos, una interpretación de atributo nominal asimilable a la predicación secundaria.

4 Según Cifuentes Honrubia / Tornel Sala la oración La niña creció rápida es agramatical (1996: 37). Aunque puede que sea así, sigue siendo cierto que el tipo de construcción se observa en el habla oral espontánea, e incluso, a veces, en el discurso feminista (Hummel, 2000:373-374). 
Observemos de entrada que la tradicional distinción ortográfica entre el adjetivo solo y el adverbio sólo fue una simple convención con la que se impuso la convicción erudita de la existencia de las categorías adjetivo y adverbio a un atributo que manifiestamente no las distingue, ya que en el habla oral no se pronuncia el acento. Se trata simplemente de uno de los muchos atributos monocategoriales que se resistieron al aluvión de los adverbios en -mente, y que mantuvieron una función adverbial al lado de la adjetival ${ }^{5}$. Como en el caso de junto, los adverbios en -mente creados por la norma culta, solamente y juntamente, empezaron a competir con ellos, presentándose, según el contexto, como alternativas de registro, o con diferencias semánticas, como, por ejemplo, en el caso de muchos adverbios oracionales del tipo igual/igualmente. Los matices diasistemáticos de su uso son tan importantes que resulta imposible tratarlos exhaustivamente a partir de los pocos ejemplos de nuestro corpus. Habría que dedicarles estudios monográficos para captar su riqueza funcional y expresiva en los ejes diacrónico y sincrónico.

En lo que concierne el uso de solo como atributo flexional en el sintagma verbal, podemos citar nueve ejemplos de nuestro corpus:

(36) pero solo porque yo me daba cuenta que me fijando en que:, como habla la gente y: yo .. he tratado de .. de hacerlo sola (Marisa, 345)

(37) creo que:, aprender a hacer lah cosah sola ...(1) sin la ayuda de nadie [...] Sí ... creo que sí ... aprender a hacer yo lah cosas sola (Irina, 106)

(38) Tener que dejarlo en=un colegio $/$.. y nunca (puedo) decirlo lah horah ... de salida del trabajo de uno-... a la salida de loh niñoh, que (encargarse)+ una vecina o que el niño se vaya solo - (Sandra, 866)

(39) entonces mi hijo vive ahí en el departamento, äh .. va a clase y se hace todo solo, ya .. se cocina, se lava solo sí .. se atiende solo [...] él vino solo a hacer preuniversitario aquí en santiago no=máh (Marta, 73).

(40) Un taller/ .. n- $=\mathrm{nO}$, pero, yo sola .. yo sola .. sí ... yo sola .. proseguir trabajo y:, trabajar yo sola .. sí (Marta, 1163)

En estas citas solo actualiza claramente una función de atributo del sujeto con el significado 'sin compañia', con excepción del primer ejemplo en el que, por casualidad, aparece también solo como adverbio (pero solo porque...). La situación de solo se complica por la competencia de solamente, que pertenece al polo bicategorial ( 5 ocurrencias en el corpus). El resultado de esta competencia es bastante complejo, en la medida en que hay que estar atento a diferencias de uso muy sutiles de orden pragmático-estilístico. Por supuesto, solamente no puede sustituir a solo con función de predicado secundario, como en nuestros ejemplos, pues éste mantiene su función de adjetivo, aunque sí es conmutable en pero solo porque... Trataremos el caso de solo / solamente con función de adverbio en otra ocasión junto con los adverbios enunciativos.

A diferencia de solo, el uso de junto está casi gramaticalizado en el sentido de que incluso la forma flexional juntos suele ser intercambiable, semánticamente, con juntamente. En nuestro corpus se encuentran dos ejemplos:

5 Comp. formas como otramente, mismamente, siquieramente que surgieron históricamente para caer en desuso después, al menos en la lengua estándar. Se observa el mismo fenómeno en francés con presquement, mêmement, vitement, etc., que desaparecieron del estándar culto, aunque sí se mantuvieron a veces en algunas variedades subestándar (Hummel en prensa). 
(41) dehpué se fueron para=allá porque ya no=no- .. aquí hay (maloh vi:cio lo sabe), en ese sentido ... dehpué el grande, el mayor se casó entonce ahora- .. viven loh dOh juntoh (Marisa, 109)

(42) son mih compañerah, pero: ... amigah amigah .. nO, o=sea, que conversamo así: .. pero, de-, ah, de=de salir $j U: N t a h$, no .. no, $o=$ sea el tiEmpo parece que no=no=no-, no permI:te (Marta, 1131)

La conmutabilidad semántica con juntamente podría indicar que junto tiende a ser adverbio a pesar de mantener la flexión, como en los ejemplos del § 7. Citemos también un ejemplo sacado del correo electrónico de un colega venezolano: "Para mí una cosa y otra, juntas o separadamente, son ustedeo, lo mismo que tuteo es el uso del pronombre o del verbo, juntos o separados, de segunda persona del singular".

Pese a su proximidad semántica, tenemos la impresión que el uso de juntamente resulta algo artificial. Seco (1998:270) observa que "el uso de junto, como adverbio (Déjalo allí junto), es propio de la lengua popular", sin referirse al problema de su flexión. De otro lado, usar sistemáticamente solamente y juntamente es señal de un estilo hipercorrecto, pedante, casi feo. Además, parece que la conciencia lingüística puede variar según la persona, la región y otros factores. La lingüista mexicana Arjona Iglesias (1991:31), por ejemplo, afirma que todos los adverbios en -mente observados en el habla popular mexicana podrían haber aparecido también en el habla culta, "excepto juntamente, a mi entender", puesto que se trataría de una forma popular. No disponemos de datos suficientes para analizar más en profundidad el caso complejo de junto (variable, invariable) frente a juntamente. Sea como fuere, el uso de juntamente lleva una marca de registro que impide intercambiarlo libremente con junto, a pesar de su proximidad semántica (comp. Hummel, 2000: 412).

Podemos concluir que lexemas como solo y junto conforman un microcosmo sintáctico, semántico y pragmático muy complejo, en el que se manifiestan en un solo lexema todas las tendencias descritas en los $\S 6$ y $\S 7$ : la invariabilidad, la flexión lógica, la flexión ilógica (a los ojos del hablante culto) y la competencia con las formas en -mente. La hipótesis de la preferencia del latín hablado por el sistema monocategorial y de su colisión ulterior con el sistema bicategorial de la norma culta escrita explica, a nivel teórico, la compleja actualidad de uso de una serie de atributos altamente frecuentes en el habla oral informal a la que pertenecen también solo y junto. Por supuesto, habría que indagar más en las peculiaridades del desarrollo histórico de cada una de estas unidades. Aquí nos limitamos a formular el marco teórico para la investigación histórica concreta.

\section{Otras fronteras categoriales borrosas: las construcciones copulativas y los adjetivos destacados}

Resulta dificil separar la predicación secundaria de las construcciones copulativas en los llamados verbos semicopulativos: La encuentro / veo feliz. Encontrar y ver son verbos polisémicos que admiten una interpretación concreta 'ubicar' / 'percibir con los ojos' y una interpretación metafórica 'opinar' / 'pensar que alguien es asi'. En su interpretación concreta, se trata de verbos plenos acompañados por un predicado secundario en función de adjunto libre, mientras que el significado metafórico los transforma en verbos copulativos que tienen el atributo como complemento obligatorio. Trataremos los detalles de estas 
construcciones en otra ocasión, aunque algunas construcciones con dejar, encontrar, ver, quedar, etc., en las que estos verbos funcionan como verbos plenos, podrían entrar aquí como predicación secundaria.

A diferencia de la expresión escrita, que puede usar o no la coma, la estructura de los enunciados orales torna prácticamente imposible distinguir la predicación secundaria de los adjetivos destacados que vienen antes o después del verbo:

(43a) María habla contenta.

(43b) María habla, contenta.

Lo único que podemos decir es que, en la oralidad, el hablante tiene la posibilidad de desprender en mayor o menor medida el atributo de la sintaxis de los constituyentes inmediatos, utilizando para este fin recursos como la pausa, la entonación y el alejamiento sintáctico del verbo, que se ven en muchos de nuestros ejemplos sacados del Corpus Kluge. Por eso, hemos optado por no distinguir formalmente los adjetivos destacados de los predicados secundarios plenamente integrados en el sintagma verbal. De hecho, son indistinguibles en la oralidad espontánea.

\section{Lógica categorial y borrosidad fenomenológica}

Desde el punto de vista funcional, la borrosidad categorial y la heterogeneidad fenomenológica documentada arriba se explica por su origen en un sistema monocategorial. El sistema monocategorial permite el uso de la misma categoría atributiva en distintas posiciones y funciones sintácticas: pagar poco, poco importante, poco vino, pocas casas. ${ }^{6} \mathrm{Si}$ la categoria atributiva es potencialmente flexional, como en las lenguas románicas, los morfemas flexionales se juntan al atributo durante la dinámica de actualización en el proceso enunciativo. E1 atributo queda invariable o flexionado según el caso: ellos pagan poco, poco importantes, pocas casas. Para el hablante, el sistema está muy claro, funcionalmente. Dispone de un sistema monocategorial que llamamos atributo, sin distinguir, a nivel categorial, entre adjetivo y adverbio, pero con la opción de usar los morfemas de flexión en el enunciado para dirigir el alcance atributivo hacia un sustantivo. La espontaneidad de este proceso explica el uso vacilante de los morfemas flexionales en el habla informal, y muchas veces también en los textos antiguos de las lenguas románicas, que carecían todavía de una normalización rigurosa. El carácter 'popular' del habla informal de nuestro corpus consiste en haber mantenido también la parte 'ilógica', donde las reglas normativas exigen al hablante culto ser lógico, buscando además una lógica más gramatical (acuerdo gramatical) que semántica (acuerdo ad sensum). Por consiguiente, el habla culta se presenta como más lógica, mientras que las vacilaciones eliminadas quedan tachadas de 'populares'. Por la misma razón, resulta más difícil separar la predicación secundaria de los adjetivos adverbializados en nuestro corpus popular que en el habla culta escrita. En nuestro análisis de 22 obras literarias (Hummel, 2000), por ejemplo, dicha separación no fue dificil. Podemos concluir que la borrosidad categorial es más fuerte en el habla informal de nuestro corpus que en un texto escrito literario o normativo (periódicos, etc.)

6 Salazar García (2007:316) propone el término "modificador flexible" cuando adjetivos y adverbios se confunden en una sola categoría. 
a causa del comportamiento lingüístico de los hablantes cultos que eliminan las incoherencias lógicas en la expresión escrita con su mayor grado de planificación y control.

El habla 'popular' hace simplemente uso de todas las posibilidades que se ofrecen en dicha posición sintáctica. Usa los morfemas flexionales como en la predicación secundaria canónica. Usa los adjetivos adverbializados como correr rápido analizados en Hummel 2007. $\mathrm{Y}$ usa lúdicamente los morfemas flexionales en camina firmadita. Renuncia a la fiexión cuando no le parece necesario en calladito obedecer. Y no le molesta seguir mecánicamente la analogía fonética en vamos directos, que es normativa en los caminos directos. Se observan todas las variantes y variedades de uso del sistema monocategorial. Es impropio, pues, hablar de 'borrosidad categorial' en estos casos. No puede haber borrosidad si se trata de una categoría única que nosotros, herederos de la tradición culta, intentamos en vano dividir en subcategorías según nuestros criterios. Por eso, hemos optado por usar las comillas en los términos predicación secundaria 'auténtica', 'semántica' y 'falsa'. Los fenómenos que acabamos de enumerar son variantes morfológicas, sintácticas y semánticas del uso de una sola categoría morfológica. A diferencia del adverbio en -mente en posición adverbal, el uso del atributo monocategorial flexional produce una variedad importante de esquemas morfo-sintáctico-semánticos: atributo del verbo invariable (o con flexión 'popular'), atributo de sujeto u objeto condicionado por el verbo, uso expresivo de los morfemas de flexión. La predicación secundaria es el esquema más complejo en el conjunto de las funciones atributivas observables, en la medida en que combina la pertenencia sintáctica del atributo a un verbo con un alcance atributivo dirigible morfológica y/o semánticamente hacia el sujeto o el objeto del verbo. Ello nos obliga a hablar de un esquema morfológico-sintáctico-semántico. Con vistas a su complejidad, ya no nos sorprende la borrosidad fenomenológica que lo caracteriza en el habla oral espontánea.

En la oralidad espontánea semiculta de nuestro corpus, los hablantes siguen percibiendo la proximidad categorial de adjetivos y adverbios, en la medida en que no les está vedada la coordinación sintáctica, como en la siguiente construcción copulativa:

(44) lo (van a) pasar bien: .. todo=lo van a pasar bien y XX feli(z), pienso yo (Julia, 901)

Semánticamente, el atributo feliz tiende a funcionar como atributo del sujeto. En este ejemplo, sin embargo, feliz rechaza la marca de plural felices, a la vez que viene coordinado sintácticamente con el adverbio léxico bien. A pesar de estas marcas fuertes que apuntan hacia una interpretación adverbial, el oyente puede interpretarlo como característica de los participantes, al menos en mayor grado que en el caso de bien. Otra vez, feliz no es ni adverbio ni adjetivo, sino un simple atributo indiferenciado cuya mira atributiva depende de la interpretación semántica y pragmática del enunciado. Mencionemos también la construcción paralela del adverbio bien con un predicado secundario orientado hacia el objeto en el ejemplo (13): "no dicen bien lah cosah [...] no la dicen:, tan:, [exact(ah)]". Mirándolo así, podriamos preferir la clasificación del ejemplo con la flexión ilógica del $\$ 7$. Por otro lado, también es posible interpretar la forma exactas como atributo de la (= las cosas). El esquema propuesto en $\S 1$ formaliza la ambivalencia que caracteriza el español actual, dudando entre el sistema monocategorial y el bicategorial, con preferencias claramente distintas entre la norma culta y el habla inculta, sin perder la conciencia de su proximidad categorial a la hora de coordinar sintácticamente sus unidades. 


\section{Un sistema monocategorial con tres tendencias}

Hemos visto que las variantes y vacilaciones atributivas producibles y producidas por el sistema atributivo monocategorial son el resultado de las opciones morfológicas, sintácticas y semánticas que se le ofrecen al hablante en la enunciación. Llegados a este punto, cabe diferenciar nuestra hipótesis de una competencia de dos sistemas de formación de adverbios en las lenguas románicas, uno monocategorial, otro bicategorial. De hecho, existen varias tendencias dentro del sistema monocategorial: una que tiende hacia la invariabilidad del atributo, como se da sistemáticamente en alemán y ocasionalmente en español, aun cuando lógicamente habría que flexionar ((ella) calladito obedecer, ella habla cantadito), y otra que tiende a realizar la flexión siempre que exista una relación atributiva con un sustantivo (ella llega cansada) o siempre que el hablante la desee expresar o subrayar (camina firmadita). La convivencia de estas dos tendencias explica el uso vacilante de la flexión.

Podemos incluso idear una tercera tendencia: la de la flexión en todos los casos, aun cuando la función atributiva es claramente adverbial, como en los casos documentados en el $\S 7$ bajo el rubro de predicación secundaria 'falsa'. El italiano, por ejemplo, tiende a la flexión general, sobre todo en los dialectos meridionales, pero también en el habla culta, quizás con menos vigor en el habla estándar no literaria (periódicos, ensayos, etc.; v. Hummel, 2000:434-440). Mencionemos algunos ejemplos que se traducirían al español sin flexionar porque, semánticamente, no hay ninguna relación atributiva con el sujeto:

(45) le sue lagrime corsero più facili. (Meyer-Lübke, 1974:448)

(46) Siamo passati veloci nell'androne [...] (De Carlo, 1995:77)

(47) Siamo scesi senza dire niente, sgusciati rapidi [...] (De Carlo, 1995:82)

(48) mi ha baciato rapida sulle labbra [...] (De Carlo, 1995:104).

En otras palabras, parece que se generalizó en italiano el tipo vamos directos, que se conoce en español aunque se usa mucho menos.

En términos cuantitativos, el grupo de las predicaciones secundarias auténticas predomina claramente en nuestro corpus, con 20 predicados secundarios orientados hacia el sujeto (incluyendo los ejemplos de solo), 4 orientados hacia el objeto y 2 con verbo reflexivo. La predicación secundaria semántica, difícilmente separable del grupo de los adjetivos adverbializados, presenta 11 casos, y la predicación secundaria 'falsa' 3 casos. Los ejemplos de junto constituyen un caso aparte, ya que se podrían clasificar tanto en el primer grupo como también en el último. Podemos concluir que el español tiende mucho menos que el italiano a la flexión 'ilógica' o 'expresiva' del atributo. A pesar del problema adicional de la elisión frecuente del morfema de plural en nuestro corpus, las hablantes tienden a flexionar el atributo de acuerdo con su relación semántica atributiva. Se favorece la invariabilidad del predicado secundario en posiciones que lo alejan del verbo y cuando se emplea con sentido genérico, por ejemplo, con las personas, la gente, etc. En total, nuestro corpus contiene 42 predicados secundarios, sin contar el ejemplo (44), que pertenece a las construcciones copulativas no tratadas aquí. Si lo relacionamos con la ocurrencia de tan sólo 5 adverbios en -mente como miembros del sintagma verbal (atributos del verbo; v. §3), podemos concluir que los predicados secundarios aumentan el peso del sistema atributivo monocategorial en 
el habla oral semiculta ${ }^{7}$. Se puede decir que en este registro recurre casi exclusivamente el atributo monocategorial, flexionándolo o no según el caso.

\section{La intervención de la norma escrita en el establecimiento diacrónico de la predica- ción secundaria como esquema (re)conocido}

Se escribe poco sobre la intervención del código en la diacronía de las construcciones gramaticales. Nuestros datos nos llevan a suponer que el establecimiento de la predicación secundaria como esquema usual en la lengua española y otras lenguas románicas está correlacionado con la tradición escrita a dos niveles: las condiciones generales propias de la expresión escrita, como el mayor grado de planificación, y su vinculación con el habla culta. A diferencia del habla oral informal, donde la predicación secundaria emerge apenas de las vacilaciones que acompañan el uso del atributo marcado por la rapidez y espontaneidad de su flexión, el habla culta tiende a separar claramente el adjetivo del adverbio, según se enseña en la escuela, sobre todo en el código escrito, que favorece la flexión lógica y gramatical por su mayor grado de planificación morfológica y sintáctica. Incluso en el caso de la predicación secundaria 'semántica', que no permite una separación formal de los adjetivos adverbializados, el habla culta escrita logra un mayor grado de clareza categorial, en la medida en que el uso sistemático de los adverbios en -mente para los atributos del verbo implica indirectamente que el uso del adjetivo junto al verbo se debe interpretar, en la expresión culta, como predicación secundaria, y no como adjetivo adverbializado, incluso cuando la invariabilidad se debe al sistema de la lengua $(\$ 6)^{8}$. Tampoco se observa en los textos escritos cultos la elisión de la $-s$ final. Los casos en los que no funciona la separación categorial son conocidos como lista casi cerrada de adjetivos adverbializados admitidos en el habla culta, tales como hablar alto, respirar hondo, etc. De ello se desprende que el lector de un texto escrito culto sabe casi siempre cuándo un atributo que aparece dentro del sintagma verbal es un adverbio y cuándo un adjetivo dirigido a un participante del verbo.

Pensamos además que el género literario de la novela y su discurso narrativo, que se establece progresivamente a partir de finales del siglo XVIII, contribuyó de forma decisiva al arraigo de la predicación secundaria. Desde el punto de vista sincrónico se puede constatar que la predicación secundaria aparece muy poco, si es que aparece, en los textos técnicos, científicos, periodísticos y en ensayos intelectuales, mientras que abunda en las novelas. El análisis semántico de la predicación secundaria en las novelas nos permite afirmar que esta construcción sirve, en primer lugar, para describir el estado mental de los personajes en el momento de actuar, sobre todo a la hora de hablar (tipo contestó tímida). En las novelas del autor realista portugués Eça de Queirós, inspiradas por Flaubert, el $57 \%$ de los atributos en predicación secundaria expresan un estado psíquico o psicofísico de una persona (Hummel, 1998a). Del mismo modo, en el corpus de 22 novelas españolas contemporáneas analizadas en Hummel 2000, el $62 \%$ de los predicados secundarios caracterizan un estado psíquico del sujeto en el momento de actuar (2000:311). Si es cierto que la novela fue el primer género

7 En su análisis de un texto literario escrito en argot francés, Kofler (2007: 21) observa también una frecuencia relativamente alta de los adjetivos adverbializados y de la predicación secundaria.

8 La argumentación de De Miguel / Fernández Lagunilla (2004) va en el mismo sentido, aunque no toman en consideración los adjetivos adverbializados. 
literario que se dedicó a la descripción sicológica de los personajes, parece lógico suponer que la predicación secundaria, cuya función es la descripción del estado del participante en el momento de actuar, se arraigó sobre todo en la novela, al mismo tiempo que se explica su escasa frecuencia en los textos técnicos, etc. mencionados arriba.

Los datos obtenidos por Alarcón Neve (2005) a partir del discurso narrativo de alumnos mexicanos confirman nuestra hipótesis desde el punto de vista del desarrollo individual de la capacidad lingüística. De entrada, no parece casual el título del trabajo, que alude directamente a la correlación de la expresión de un estado mental con el discurso narrativo: "Estrategias lingüísticas para la descripción de los estados mentales dentro del discurso narrativo de los niños en edad escolar: adjetivos en predicación". De manera general, la casi totalidad de los adjetivos que expresan un estado mental (348 casos de un total de 351) aparecen en función predicativa, sea con verbos copulativos u otros, frente a tan sólo 3 adjetivos adnominales (2005: 76-77). Dentro de los atributos en función predicativa se observa un predominio de las construcciones copulativas, que llevan el adjetivo como complemento, en los alumnos más jóvenes ( 6 años de edad, $1^{\circ}$ grado), especialmente de estar. El uso de los adjetivos en otras construcciones copulativas aumenta con el grado de formación escolar, que fue observado hasta la edad de 12 años ( $5^{\circ}$ grado), igual que el de los adjetivos en predicación secundaria y de los adjetivos destacados (2005: 85). De ello se desprende que el uso de los adjetivos como adjuntos cercanos o destacados del verbo aumenta con el desarrollo individual de la capacidad narrativa. El discurso narrativo elaborado de las novelas literarias parece haber ido por el mismo camino.

Notemos finalmente que la primera toma de conciencia lingüística inequívoca del fenómeno data del siglo XIX del francés Ferdinand Brunot (1969: 361). Hasta bien entrado el siglo XX, la predicación secundaria no aparece en las gramáticas del francés como fenómeno claramente distinto de las construcciones copulativas, por un lado, y de los adjetivos adverbializados, por otro (Hummel en prensa c). Hubo que esperar hasta la segunda mitad del siglo XX para que se manifestara una conciencia general en la lingüistica de la existencia de esta función atributiva. Es decir, la aparición tardía de la predicación secundaria en la conciencia de los lingüistas y autores de gramáticas confirma que se trata de una construcción que se estableció tarde en la diacronía de las lenguas románicas, al menos en términos de frecuencia, aunque existió desde sus principios como variante espontánea. Por supuesto, habría que confirmar esta hipótesis mediante el análisis de un corpus diacrónico compuesto por diferentes tipos de texto.

\section{Conclusión}

Diacrónicamente, la predicación es una construcción que, si bien nació en la tradición oral, se estableció como esquema usual a través de los textos escritos, posiblemente sobre todo en el género narrativo psicologizante de la novela decimonónica. Resulta interesante constatar el papel de la expresión escrita en el nacimiento de un esquema gramatical, consecuencia directa del mayor grado de planificación y coherencia lógicas del habla escrita culta, frente a las variantes y vacilaciones del habla oral informal, consecuencias de su rapidez, espontaneidad y aspecto lúdico, y tachadas de 'populares' por la norma culta. Por supuesto, podría argüirse que la predicación secundaria nacería también si existiera una categoría morfológica del adjetivo claramente separada de la del adverbio. Pero, en tal caso, no existiría 
la borrosidad. Por un lado, los adjetivos adverbializados se extienden formalmente como adverbios flexivos hacia la función adjetival (tipo vamos directos a casa), por otro lado, la predicación secundaria semántica crea sintagmas que, pese a la invariabilidad del atributo, se refieren al sujeto u objeto del verbo (tipo calladito obedecer). Este hecho se explica por la existencia de una sola categoría morfológica que cumple tanto las funciones de adjetivo como las de adverbio. Las 42 predicaciones secundarias de nuestro corpus aumentan la importancia del sistema atributivo monocategorial en lo que atañe a su uso dentro del sintagma verbal, puesto que sólo se encuentran 5 adverbios en -mente como miembros de un sintagma verbal en todo el corpus, frente a 82 atributos monocategoriales, si adicionamos los 40 adjetivos adverbializados documentados en Hummel 2007. Desde el punto de vista de la lógica funcional del sistema monocategorial, deberíamos haber tratado los dos grupos en una sola contribución.

\section{Referencias bibliográficas}

Alarcón Neve, L. (2005): Estrategias lingüisticas para la descripción de los estados mentales dentro del discurso narrativo de los niños en edad escolar: adjetivos en predicación, Memoria de investigación bajo la dirección de Vidal Lamíquiz Ibáñez. Madrid, UNED.

Brunot, F. (1969) [= 1891]: La Doctrine de Malherbe d'après son commentaire sur Desportes. Paris, Colin.

Carlo, A. de (1995): Due di due. Milano, Mondadori.

Cifuentes Honrubia, José Luis y José Luis Tornel Sala (1996): "El predicativo en español: iconicidad y gramática", Lingüistica española actual, 18,1, págs. 17-47.

De Miguel, E. y M. Fernández Lágunilla (2004): "Un enfoque subeventivo de la relación entre predicados secundarios y adverbios de manera", Revue Romane, 39,1, págs. 24-44.

González García, L. (1997): El adverbio en español. A Coruña, Universidade da Coruña.

Hummel, M. (1998a): "Adverbale Adjektive in Os Maias von Eça de Queirós". En Hummel, Martin y Christina Ossenkop (dirs.): Lusitanica et Romanica, Festschrift für Dieter Wọll. Hamburg, Buske, págs. 153-176.

Hummel, M. (1998b): "Zur Übersetzung adverbaler Adjektive aus dem Spanischen ins Französische. Mit einem kurzen Blick auf das Italienische und Portugiesische". En Figge, Udo. L., Franz-Josef Klein, Annette Martinez Moreno (dirs.): Grammatische Strukturen und grammatischer Wandel. Festschrift für Klaus Hunnius zum 65. Geburtstag. Bonn, Romanistischer Verlag, págs. 209-233.

Hummel, M. (2000): Adverbale und adverbialisierte Adjektive im Spanischen. Konstruktionen des Typs Los niños duermen tranquilos und María corre rápido. Tübingen, Narr.

Hummel, M. (2001): “Adjetivos adverbales flexionados y adjetivos adverbializados invariables en castellano contemporáneo. Construcciones del tipo Los niños duermen tranquilos y Maria corre rápido", Lengua 12 (Universidad Mayor de San Andrés, La Paz), págs. 9-52.

Hummel, M. (2002a): "Considerações sobre os tipos Ela fala esquisito e Ela chega cansada no portum guês coloquial e literario do Brasil e de Portugal", Confluência 24 (Rio de Janeiro), págs. 43-70.

Hummel, M. (2002b): "Sincronía y diacronía del sistema atributivo de las lenguas románicas", Lengua 13 (Universidad Mayor de San Andrés, La Paz), págs. 9-48.

Hummel, M. (2007): "Adjetivos adverbializados y otros atributos directos del verbo en el habla oral informal de Chile",. En Cuartero Otal, Juan y Martina Emsel (dirs.), Vernetzungen. Bedeutung in Wort, Satz und Text. Festschrift für Gerd Wotjak zum 65. Geburtstag, vol. 1. Frankfurt am Main, etc., Peter Lang, págs. 221-233.

Hummel, M. (en prensa a): "La función atributiva cuantitativa en el habla oral informal de Chile".

Hummel, M. (en prensa b): "Los adverbios de tiempo y de lugar en el habla oral informal de Chile". 
Hummel, M. (en prensa c): "La diachronie du système adverbial des langues romanes: tradition orale et tradition écrite". En Actes du XXVe Congrès International de Linguistique et de Philologie Romanes. Innsbruck 2007.

Karlsson, K. E. (1981): Syntax and affixation. The evolution of MENTE in Latin and Romance. Tübingen, Niemeyer.

Kluge, B. (2005a): Identitätskonstruktion im Gespräch: südchilenische Migrantinnen in Santiago de Chile. Frankfurt / Madrid, Vervuert / Iberoamericana.

Kluge, B. (2005b): "Las fórmulas de tratamiento en un corpus chileno". En Noll, Volker, Klaus Zimmermann, Ingrid Neumann-Holzschuh (dirs.): El español en América: Aspectos teóricos, particularidades, contactos. Frankfurt / Madrid, Vervuert / Iberoamericana, págs. 169-188.

Kofler, M. (2007): Der Gebrauch der Modaladverbien in drei französischsprachigen Romanen von Andreï Makine, Philippe Djian und Alphonse Boudard. Tesina, Graz, Karl-Franzens-Universität.

Meyer-Lübke, W. (1974) [ $=1890-1906]$ : Grammaire des langues romanes, traduite par Auguste et Georges Doutrepont, vol. 3: Syntaxe. Genève / Marseille, Slatkine / Laffitte.

Salazar García, V. (2007): "Flexibilidad categorial y adverbios de manera en español: un enfoque funcional". En Barros García, Pedro, Gonzalo Águila Escobar, Esteban Tomás Montero del Arco (dirs.): Estudios lingüísticos, literarios e históricos. Homenaje a Juan Martínez Marín. Granada, Universidad de Granada, págs. 309-326.

Seguí, A. F. (1992), "Das spanische Prädikativ", Wissenschaftliche Zeitschrift der Humboldt-Universität zu Berlin, Reihe Geistes- und Sozialwissenschaften, 41,3, págs. 85-92. 